\title{
Sulforaphane Potentiates Anticancer Effects of Doxorubicin and Cisplatin and Mitigates Their Toxic Effects
}

\author{
Cinzia Calcabrini, Francesca Maffei, Eleonora Turrini and Carmela Fimognari ${ }^{\star}$ \\ Department for Life Quality Studies, Alma Mater Studiorum-Università di Bologna, Rimini, Italy
}

The success of cancer therapy is often compromised by the narrow therapeutic index of many anticancer drugs and the occurrence of drug resistance. The association of anticancer therapies with natural compounds is an emerging strategy to improve the pharmaco-toxicological profile of cancer chemotherapy. Sulforaphane, a phytochemical found in cruciferous vegetables, targets multiple pathways involved in cancer development, as recorded in different cancers such as breast, brain, blood, colon, lung, prostate, and so forth. As examples to make the potentialities of the association chemotherapy raise, here we highlight and critically analyze the information available for

OPEN ACCESS

Edited by:

Katrin Sak,

NGO Praeventio, Estonia

Reviewed by:

Frederick E. Williams,

University of Toledo, United States

Marco Falasca,

Curtin University, Australia

${ }^{*}$ Correspondence:

Carmela Fimognari

carmela.fimognari@unibo.it

Specialty section:

This article was submitted to Pharmacology of Anti-Cancer Drugs, a section of the journal

Frontiers in Pharmacology

Received: 22 December 2019

Accepted: 14 April 2020

Published: 01 May 2020

Citation:

Calcabrini C, Maffei F, Turrini E and Fimognari C (2020) Sulforaphane

Potentiates Anticancer Effects of Doxorubicin and Cisplatin and Mitigates Their Toxic Effects.

Front. Pharmacol. 11:567. doi: 10.3389/fphar.2020.00567 two associations, each composed by a paradigmatic anticancer drug (cisplatin or doxorubicin) and sulforaphane.

Keywords: sulforaphane, doxorubicin, cisplatin, anticancer effects, Nrf2, chemosensitization, chemoresistance, toxicity

\section{INTRODUCTION}

A promising strategy to improve the efficacy of anticancer therapy is the association of chemotherapeutic drugs with natural compounds (Farzaei et al., 2016; Negrette-Guzman, 2019). Indeed, in tumor tissues, phytochemicasl may interact with multiple molecular targets and potentiate the efficacy of traditional anticancer drugs. Moreover, they might exert a protective role against side effects caused by chemotherapeutic agents on off-target tissues.

Sulforaphane (SFN) is a natural isothiocyanate extensively studied for its pleiotropic activity on different cancer models. SFN has been found to exhibit cytotoxic and cytostatic activities through several mechanisms. The production of reactive oxygen species (ROS) is one of the most important. SFN-induced ROS generation promotes the activation of both intrinsic and extrinsic apoptotic pathways. SFN can also cause cell-cycle arrest in tumor cells, partly dependent on the modulation of epigenetic mechanisms including histone acetylation and DNA methylation (Brioness-Herrera et al., 2018). Its activity has been reported even in the most advanced stages of cancer development, where it inhibits pathways involved in metastasis and angiogenesis (Sestili and Fimognari, 2015; Negrette-Guzman, 2019). A very recent study reported that the anticancer activity of SFN involves microRNAs (miRNAs) regulation. miRNAs are post-transcriptional regulators of genes implicated in critical cellular pathways, including apoptosis, cell cycle, and cell differentiation (Rafiei et al., 2020).

A peculiar characteristic of SFN is its ability to exert dichotomous effects. Indeed, SFN is also an indirect ROS scavenger: it up-regulates phase II biotransformation enzymes by enhancing Nuclear factor E2-related factor 2 (Nrf2) activity. SFN disrupts the link between Nrf2 and its suppressor 
Kelch-like ECH-associated protein 1 (Keap1) and promotes the cytoplasmic and nuclear accumulation of Nrf2 (Briones-Herrera et al., 2018). In the nucleus, Nrf2 acts as a transcription activator for DNA sequences known as antioxidant response elements (ARE). SFN via Nrf2 increases the expression of some AREtarget genes including $\mathrm{NADPH}$-quinone oxidoreductase 1 (NQO1), heme-oxygenase (HO-1), and glutamate-cysteine ligase catalytic subunit (GCLC).

In this mini-review, we highlight and critically analyze the available evidence on the anticancer and cytoprotective effects of $\mathrm{SFN}$ in association with two paradigmatic anticancer drugs, i.e., doxorubicin (Doxo) and cisplatin (CIS).

\section{SFN AND DOXO ASSOCIATION}

\section{SFN Enhances the Anticancer Efficacy of Doxo}

The anthracycline Doxo induces DNA damage through different mechanisms such as topoisomerase II inhibition, generation of ROS, and DNA adduct formation. Doxo undergoes bioreductive activation by redox-cycling reactions, forming a reactive semiquinone. The semiquinone radical intercalates in DNA duplex and generates ROS. ROS increase DNA damage resulting in cytotoxic and cytostatic events (Agudelo et al., 2014). Of note, the generation of ROS is a double-edged sword. It is the key mechanism through which Doxo induces tumor cell death but, at the same time, it may contribute to Doxo toxicity (Angsutararux et al., 2015; Karasawa and Steyger, 2015) and prompt signals leading cancer cells to escape apoptosis (Alimbetov et al., 2018).

In combination with Doxo, SFN increased its proapoptotic activity in different cell lines (Fimognari et al., 2012; Bose et al., 2018; Mielczarek et al., 2019) (Figure 1). Furthermore, SFN reverted Doxo-resistant phenotype in p53-mutated cells, inducing apoptosis irrespective of p53 status (Fimognari et al., 2006; Fimognari et al., 2007). SFN potentiated also the RNAdamaging activity of Doxo, increasing its proapoptotic potential (Fimognari et al., 2012). Besides, SFN improved the sensitivity to Doxo by inducing autophagy via epigenetic mechanisms. In particular, SFN suppressed histone deacetylase HDAC6 that in turn activates PTEN (phosphatase and tensin homolog), a tumor suppressor gene and key regulator of autophagy (Yang F, et al., 2018). However, in certain cancer cell lines, SFN showed a hormetic biphasic response. At low doses, it reduced Doxoinduced oxidative stress, but at higher doses it exhibited synergistic effects and promoted DNA damage (Zanichelli et al., 2012) (Figure 1).

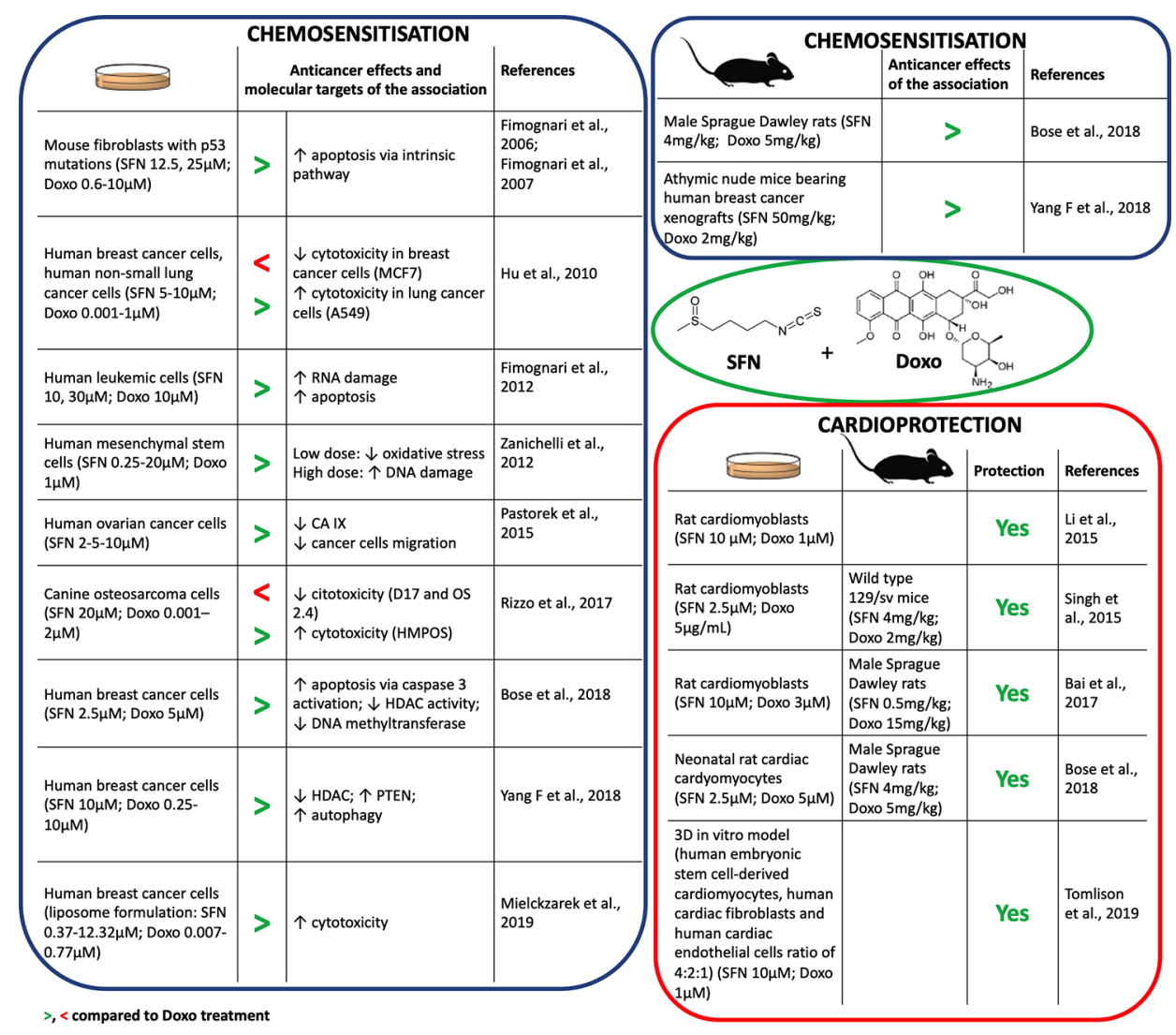

FIGURE 1 | Chemosensitization and cardioprotection of sulforaphane (SFN) in association with doxorubicin (Doxo). 
Some anticancer drugs can lose their efficacy in a hypoxic cancer microenvironment (Muz et al., 2015). The master genes orchestrating molecular response to hypoxia are HIF1 $\alpha$ (hypoxia-inducible factor $1 \alpha$ ) and its downstream targets, such as carbonic anhydrase protein IX (CA IX). CA IX protein protects from $\mathrm{pH}$ imbalance provoked by hypoxia and facilitates invasion and migration of tumor cells (Tafreshi et al., 2014). SFN down-regulated the expression of HIF1 $\alpha$ and CA IX proteins in ovarian cancer cells cultivated in hypoxia and reduced their migration (Pastorek et al., 2015) (Figure 1). Since HIF1 $\alpha$ was found to be upregulated in tumor cells after Doxo treatment (Cao et al., 2013), the down-regulation of HIF1 $\alpha$ by SFN could represent a relevant mechanism to enhance Doxo efficacy in cancer cells.

However, conflicting data on the effects of SFN when used in association with Doxo impose caution. Rizzo and coworkers showed that SFN can decrease Doxo's antitumor potential depending on the specific redox status of the cell line (Rizzo et al., 2017). SFN sensitized cells characterized by high basal Nrf2 expression to Doxo, whereas it reduced Doxo's anticancer effects in cells with very low $\mathrm{Nrf} 2$ basal levels (Hu et al., 2010) (Figure 1). Thus, the effects of SFN+Doxo may depend on the Nrf2 basal level of tumor cell type. Of note, most of the data on $\mathrm{SFN}+$ Doxo effects was obtained by in vitro studies. Evidence has started to accrue in vivo (Figure 1) and confirmed the synergistic effect of the association. The association of SFN could thus allow the use of lower doses of Doxo and a reduction of its adverse effects. Accordingly, Bose and coworkers demonstrated that the effective dosage of Doxo could be lowered by $50 \%$ in combination with SFN (Bose et al., 2018). Altogether, data on SFN-Doxo association are promising, but not conclusive.

\section{SFN Mitigates Doxo-Induced Cardiotoxicity}

The most common adverse effect in patients receiving Doxobased chemotherapy is cardiotoxicity. The mechanism of Doxo cardiotoxicity is multifactorial. It includes ROS-mediated myocardium injury, impaired mitochondrial function, cardiomyocyte apoptosis, and dysregulation of $\mathrm{Ca}^{2+}$ homeostasis. All together these events lead to an increased rate of heart failure (Bai et al., 2017; Tomlinson et al., 2019).

Several in vitro studies showed the cardioprotective effects of SFN after pre- or co-treatment with Doxo (Figure 1). SFN contrasted Doxo-induced oxidative stress and cardiomyocytes' death. In particular, SFN prevented apoptosis inhibiting: i) the activation of Bax protein, ii) the release of cytochrome $c$, iii) the activation of caspase-3, iv) the loss of mitochondrial transmembrane potential, and $v$ ) the generation of mitochondrial ROS (Li et al., 2015; Singh et al., 2015). SFN cardioprotection was mediated by Nrf2 activation and the subsequent induction of phase II enzymes, such as HO-1 ( $\mathrm{Li}$ et al., 2015). Interestingly, Tomlison and colleagues confirmed the pivotal role of Nrf2 in a 3D model exhibiting key features of cardiac tissue (Figure 1). Using this model, inducers of $\mathrm{Nrf} 2$, including SFN, exploited cardioprotective activity similar to dexrazoxane, used in patients receiving high cumulative dose of anthracyclines (McGowan et al., 2017). Similarly, SFN counteracted oxidative damage and heart failure induced by Doxo in vivo (Figure 1). In particular, SFN activated cardiac Nrf2 and upregulated its downstream targets, including genes involved in glutathione (GSH) synthesis, HO-1, and NQO1 (Singh et al., 2015; Bai et al., 2017; Bose et al., 2018). The reduction of Doxo-induced myocardial injury markers, such as creatine kinase-MB, aspartate aminotransferase, lactate dehydrogenase, and troponin I, further support the cardioprotective activity of SFN (Singh et al., 2015; Bai et al., 2017).

Doxo strongly compromised mitochondrial activity, due to its conversion by the mitochondrial complex I of the electron transport chain (ETC) into the more reactive semiquinone (Bose et al., 2018). SFN preserved ETC functionality and mitochondria ultrastructure of cardiac cells from oxidative stress damage in Doxo-treated animal models (Singh et al., 2015; Bose et al., 2018).

Fibrosis and inflammation can contribute to heart stiffness and dysfunction. SFN prevented Doxo-induced cardiac fibrosis inhibiting cardiac collagen accumulation and contrasting the upregulation of connective tissue growth factors induced by Doxo (Bai et al., 2017). Moreover, it decreased Doxo-induced inflammatory heart markers, such as plasminogen activator inhibitor-1 (Bai et al., 2017) and serum levels of IL-6 and TNF- $\alpha$ (tumor necrosis factor- $\alpha$ ) (Bose et al., 2018).

Finally, SFN led to an increased survival rate in animals cotreated with SFN+Doxo compared to those treated with Doxo ( $85 \%$ reduction in rats and $90 \%$ reduction in mice in hazard of dying from Doxo exposure) (Singh et al., 2015; Bose et al., 2018). This evidence is mainly imputable to the preservation of heart functionality (measured by ejection fraction, fractional shortening, and stroke volume) mediated by SFN.

On the whole, in vitro mechanistic studies and in vivo results univocally outline SFN as a promising molecule to prevent Doxo-induced cardiotoxicity.

\section{SFN AND CIS ASSOCIATION}

\section{SFN Enhances the Anticancer Efficacy of CIS}

CIS is a platinum derivative used for both solid and liquid cancer treatment (Volarevic et al., 2019). Its anticancer activity is due to multiple mechanisms involving binding to genomic or mitochondrial DNA to generate DNA damage and interfering with DNA repair systems, eventually leading to activation of p53 and induction of apoptosis. CIS-induced DNA damage is also due to its ability to generate ROS (Ghosh, 2019). Thus, compounds able to increase ROS or DNA damage could enhance CIS anticancer effects.

Many studies reported that SFN synergizes with CIS in counteracting cancer development (Figure 2). SFN enhanced CIS-induced DNA damage and apoptosis in many cancer cell lines (Hunakova et al., 2014; Lan et al., 2017; Lee and Lee, 2017; Elkashty et al., 2018; Kan et al., 2018; Xu et al., 2019). In most of 


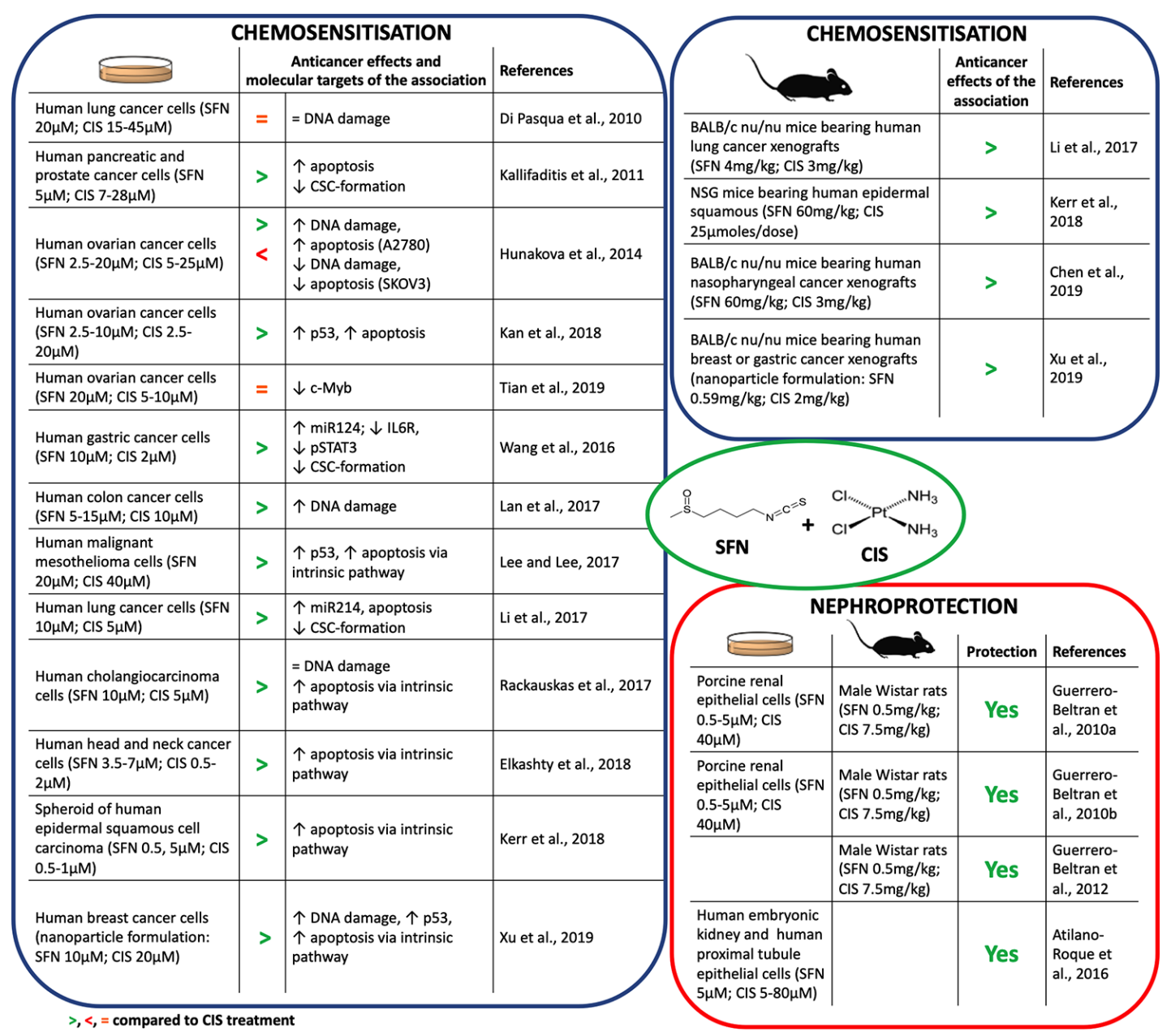

FIGURE 2 | Chemosensitization and nephroprotection of sulforaphane (SFN) in association with cisplatin (CIS).

them apoptosis occurred via p53 and caspases activation. Few studies, however, deepened the mechanisms involved in those effects. The SFN's ability to inhibit DNA repair (Piberger et al., 2014) or to transiently depletes GSH (Pappa et al., 2007) represent two candidate mechanisms. In particular, GSH depletion lowered the inactivation/excretion of CIS that occurs mainly via conjugation with GSH or metallothioneins (Ghosh, 2019). Accordingly, a nanoparticle delivery system containing SFN plus CIS decreased GSH levels and enhanced the intracellular levels of CIS (Xu et al., 2019). Since GSH depletion deprives cells of one of the most important defenses against oxidative stress, a possible consequence could be an increase in ROS and an enhanced CIS-induced DNA damage. The role of ROS generation on the cytotoxicity of SFN+CIS was defined pre-treating cancer cells with $\mathrm{N}$-acetylcysteine (NAC), a GSH precursor. NAC prevented ROS generation, activation of the mitochondrial apoptotic pathway as well as cell-cycle arrest and autophagy induced by SFN+CIS (Lee and Lee, 2017). Similarly, NAC abrogated the antitumor activity of SFN+CIS nanoparticles in vivo (Xu et al., 2019) (Figure 2).
Interestingly, SFN can increase the cytotoxicity of CIS also through mechanisms different from DNA damage. The association reduced the CIS-induced overexpression of antiapoptotic proteins such as Bcl2 (Rackauskas et al., 2017) (Figure 2), an event involved in the onset of chemoresistance (Galluzzi et al., 2012).

Another mechanism of CIS chemoresistance is the formation of cancer stem cells (CSC) (Wang et al., 2016). Both in in vitro and in vivo models, CIS-resistant cells overexpress $\beta$-catenin and c-Myc proteins, which are involved in CSC self-renewal (Li et al., 2017). CIS+SFN reduced the CSC population and inhibited their stem-like cell properties and viability in many cancer cells (Kallifaditisis et al., 2011; Wang et al., 2016; Li et al., 2017) (Figure 2). SFN reduced the activation of $\beta$-catenin/c-Myc pathway through the up-regulation of $\mathrm{miR}-214$, a negative post-translational regulator of both $\mathrm{c}-\mathrm{Myc}$ and $\beta$-catenin ( $\mathrm{Li}$ et al., 2017). Through the up-regulation of one more miRNA, i.e., miR-124 targeting the IL-6 receptor gene (Xiao et al., 2015), SFN counteracted CIS-activation of IL-6/STAT3 pathway, which seems to be involved in CIS-induced expansion of CSC cells 
(Wang et al., 2016). STAT3 signaling is also activated by c-Myb, a protein associated with CIS resistance and CSC self-renewal (Zhang et al., 2012). SFN reverted c-Myb-induced cancer cell proliferation and invasion and sensitized cells to CIS (Tian et al., 2019).

In summary, many reports disclose the ability of SFN to enhance CIS's anticancer activity and counteract chemoresistance, although there are some exceptions. As an example, SFN did not enhance CIS's cytotoxicity in a lung cancer cell line. Tubulin-binding drugs are widely used with CIS to enhance its cytotoxicity in non-small cell lung cancer. SFN, if compared with other isothiocyanates, weakly depletes $\beta$-tubulin levels (Di Pasqua et al., 2010). This evidence could explain its lack of activity in those cells. Besides, SFN exhibited a controversial role in two ovarian cancer cell lines: it synergized with CIS in A2780 cells and antagonized CIS effects in SKOV3 cells (Hunakova et al., 2014). A2780 cells have a weakly efficient Nrf2 pathway and cannot restore the depletion of GSH induced by SFN. Thus, the association significantly increased DNA damage and apoptosis compared to CIS alone. Conversely, SKOV3 cells have a highly efficient Nrf2 pathway. Thus, SFN-induced activation of the Nrf-2 pathway protected SKOV3 cells from the cytotoxicity of CIS instead of sensitizing them to CIS (Hunakova et al., 2014).

\section{SFN Mitigates CIS-Induced Nephrotoxicity}

CIS therapy causes nephrotoxicity in $30-40 \%$ of patients (Volarevic et al., 2019). The mechanism behind the onset of nephrotoxicity is particularly complex and involves multiple mechanisms, including ROS generation, mitochondrial dysfunction, apoptosis, necrosis, and autophagy of renal cells. Moreover, inflammation exacerbates these processes (Holditch et al., 2019).

ROS generation and mitochondrial dysfunction represent the earliest events in CIS-induced nephrotoxicity. SFN reduced CISinduced ROS generation in vitro. It increased GSH pool and antioxidant enzyme activity, and reduced markers of nitrosative and oxidative stress. Accordingly, SFN ameliorated cellular, plasma, kidney, and liver oxidative status (Guerrero-Beltran et al., 2010a; Guerrero-Beltran et al., 2010b; Atilano-Roque et al., 2016) (Figure 2). Furthermore, SFN improved renal histopathology and physiological functions in rats treated with CIS (Guerrero-Beltran et al., 2010a; Guerrero-Beltran et al., 2010b). SFN antioxidant activity takes place through the Nrf2 pathway. Treatment with SFN before CIS exposure activated the Nrf2 pathway and its target genes (i.e., GCLC and NQO1) and protected from CIS-induced renal cell injury (Guerrero-Beltran et al., 2010a; Atilano-Roque et al., 2016). The inhibition of GCLC and NQO1 nullified nephroprotection (Guerrero-Beltran et al., 2010a). This finding clearly points out the close link between the SFN-protective effect and its ability to activate the Nrf2 pathway.

CIS accumulates in mitochondria (Dzamitika et al., 2006) and depletes GSH levels, thus increasing mitochondrial oxidative stress and damage to complex I (Guerrero-Beltran et al., 2010a). ROS exacerbate complex I damage and activate several pathways involved in apoptosis or inflammation (Sharma et al., 2009). SFN prevented CIS-induced alterations of mitochondrial functionality in rat kidney (Guerrero-Beltran et al., 2010a) and counteracted the pathways activated by ROS in CIS-induced kidney damage (Guerrero-Beltran et al., 2012). In particular, SFN increased pro-survival ERK (extracellular signal-regulated kinase) and antiapoptotic p38 $\beta$ mitogen-activated protein kinase, and decreased the proapoptotic Jun $\mathrm{N}$-terminal kinase (JNK) and $\mathrm{p} 38 \alpha$ pathways. SFN was also able to decrease TNF- $\alpha$, nuclear factor kappa-light-chain-enhancer of activated $\mathrm{B}$ cells $(\mathrm{NF}-\kappa \mathrm{B})$, adhesive molecule expression, and leukocytes and macrophage recruitment into renal tissue and reduce kidney inflammation (Guerrero-Beltran et al., 2012) (Figure 2).

These findings highlight the pivotal role of oxidative stress in CIS toxicity and the ability of SFN of counteracting these events through its antioxidant properties.

\section{CONCLUSIONS}

Pre-clinical existing data highlight that SFN enhances the anticancer activity of Doxo and CIS and counteracts the off-target toxicity through multiple mechanisms. In particular, SFN strongly activates the Nrf2 antioxidant signaling pathway. This evidence could have clinically relevant implications for cancer therapy as Nrf2 activation in cancer cells may contribute to the onset of either chemosensitisation or chemoresistance (Bai et al., 2016; Catanzaro et al., 2017). Most anticancer drugs amplify ROS levels in cancer cells over a threshold to induce cell death and tumor regression. Anthracyclines produce the highest levels of cellular ROS; alkylating drugs, platinum-based drugs, camptothecins, arsenic-based drugs, and topoisomerase inhibitors generate high levels of ROS; taxanes, Vinca alkaloids, nucleotide analogues, and antimetabolites induce lower levels of ROS (Yang H, et al., 2018). This means that the effect of SFN when used in association with anticancer therapy could be not easily predicted, and indeed, even with the two anticancer drugs included in our review, the effects of SFN are sometimes discordant, as reported above.

In addition, it is well known that cancer cells are not homogeneous. Reprogramming of cancer cells impacts on disease's progression and contributes to their heterogeneity (Milkovic et al., 2017). As an example, in later stages of cancer, Nrf2 and Keap1 are mutated and Nrf2 activity increased. This means that inhibitors of Nrf2 could be better than activators of Nrf2 in the later stages of the disease. Thus, cancer stage should be taken into account for the usage of specific Nrf2 activators or inhibitors during cancer therapy.

Of note, Nrf2 modulation was observed in women orally treated with a broccoli sprout preparation containing $200 \mu \mathrm{mol}$ of SFN/g. In their breast tissues, increased NQO1 and HO-1 transcripts and NQO1 enzymatic activity have been found (Cornblatt et al., 2007). A phase-II clinical trial is actually recruiting patients with the aims to investigate the ability of SFN-rich broccoli sprout extracts to (i) enhance Doxo anticancer effects on women with breast cancer undergoing neoadjuvant chemotherapy, with no prior cardiac disease and who will receive Doxo without Her-2 receptor antagonists as part of their clinical care, (ii) protect from Doxo-associated cardiac dysfunction, and (iii) explore the role of $\mathrm{Nrf} 2$ in this association therapy (ClinicalTrial.gov identifier: NCT03934905, 2019). This interventional study will certainly contribute to define the role 
of Nrf2 modulation in the efficacy and safety of SFN when associated with traditional anticancer therapies.

Concerning the safety profile of SFN in association with anticancer drugs, SFN prevented changes in animal body weight caused by either Doxo or CIS (Singh et al., 2015; Kerr et al., 2018; Chen et al., 2019). However, a recent study recorded hematic signs of possible myelosuppression and hepatotoxicity in animals exposed to CIS+SFN. Interestingly, these side effects became negligible when drugs were delivered in nanoparticles (Xu et al., 2019), a formulation improving the release of drugs in tumor cells. Thus, a controlled delivery system may enhance chemotherapy efficacy and reduce systemic toxicity of SFN+CIS.

\section{REFERENCES}

Agudelo, D., Bourassa, P., Berube, G., and Tajmir-Riahi, H. A. (2014). Intercalation of antitumor drug doxorubicin and its analogue by DNA duplex: structural features and biological implications. Int. J. Biol. Macromol. 66, 144-150. doi: 10.1016/j.ijbiomac.2014.02.028

Alimbetov, D., Askarova, S., Umbayev, B., Davis, T., and Kipling, D. (2018). Pharmacological targeting of cell cycle, apoptotic and cell adhesion sinaling pathways implicated in chemoresistance of cancer cells. Int. J. Mol. Sci. 19 (6), E1690. doi: 10.3390/ijms19061690

Angsutararux, P., Luanpitpong, S., and Issaragrisil, S. (2015). Chemotherapyinduced cardiotoxicity: overview of the roles of oxidative stress. Oxid. Med. Cell Longev. 2015, 795602. doi: 10.1155/2015/795602

Atilano-Roque, A., Wen, X., Aleksunes, L. M., and Joy, M. S. (2016). Nrf2 activators as potential modulators of injury in human kidney cells. Toxicol. Rep. 3, 153-159. doi: 10.1016/j.toxrep.2016.01.006

Bai, X., Chen, Y., Hou, X., Huang, M., and Jin, J. (2016). Emerging role of NRF2 in chemoresistance by regulating drug-metabolizing enzymes and efflux transporters. Drug Metab. Rev. 48 (4), 541-567. doi: 10.1080/03602532.2016.1197239

Bai, Y., Chen, Q., Sun, Y. P., Wang, X., Lv, L., Zhang, L. P., et al. (2017). Sulforaphane protection against the development of doxorubicin-induced chronic heart failure is associated with Nrf2 Upregulation. Cardiovasc. Ther. 35 (5), e12277. doi: 10.1111/1755-5922.12277

Bose, C., Awasthi, S., Sharma, R., Benes, H., Hauer-Jensen, M., Boerma, M., et al. (2018). Sulforaphane potentiates anticancer effects of doxorubicin and attenuates its cardiotoxicity in a breast cancer model. PloS One 13 (3), e0193918. doi: 10.1371/journal.pone.0193918

Briones-Herrera, A., Eugenio-Perez, D., Reyes-Ocampo, J. G., Rivera-Mancia, S., and Pedraza-Chaverri, J. (2018). New highlights on the health-improving effects of sulforaphane. Food Funct. 9 (5), 2589-2606. doi: 10.1039/c8fo00018b

Cao, Y., Eble, J. M., Moon, E., Yuan, H., Weitzel, D. H., Landon, C. D., et al. (2013). Tumor cells upregulate normoxic HIF-1 $\alpha$ in response to doxorubicin. Cancer Res. 73 (20), 6230-6242. doi: 10.1158/0008-5472.CAN-12-1345

Catanzaro, E., Calcabrini, C., Turrini, E., Sestili, P., and Fimognari, C. (2017). Nrf2: a potential therapeutic target for naturally occurring anticancer drugs? Expert Opin. Ther. Targets 21 (8), 781-793. doi: 10.1080/14728222.2017.1351549

Chen, L., Chan, L. S., Lung, H. L., Yip, T. T. C., Ngan, R. K. C., Wong, J. W. C., et al. (2019). Crucifera sulforaphane (SFN) inhibits the growth of nasopharyngeal carcinoma through DNA methyltransferase 1 (DNMT1)/ Wnt inhibitory factor 1 (WIF1) axis. Phytomedicine 63, 153058. doi: 10.1016/j.phymed.2019.153058

ClinicalTrials.gov Identifier: NCT03934905 (2019). Protective Effects of the Nutritional Supplement Sulforaphane on Doxorubicin-Associated Cardiac Dysfunction, [Online]. Available: https://clinicaltrials.gov/ct2/show/ NCT03934905? term $=$ sulforaphane\&draw $=2 \&$ rank $=1$ [Accessed 13/12/2019].

Cornblatt, B. S., Ye, L., Dinkova-Kostova, A. T., Erb, M., Fahey, J. W., Singh, N. K., et al. (2007). Preclinical and clinical evaluation of sulforaphane for chemoprevention in the breast. Carcinogenesis 28 (7), 1485-1490. doi: 10.1093/carcin/bgm049

Di Pasqua, A. J., Hong, C., Wu, M. Y., McCracken, E., Wang, X., Mi, L., et al. (2010). Sensitization of non-small cell lung cancer cells to cisplatin by naturally
Last but not least, the stability and bioavailability of SFN may influence its chemosensitizing/chemoprotective effects. Thus, the pharmacokinetics of SFN in association with anticancer drugs should be addressed to fully understand its clinical potential in the oncological field.

\section{AUTHOR CONTRIBUTIONS}

CC, FM, ET, and CF analyzed the scientific literature. CC, FM, and ET wrote the manuscript. CF designed the study and revised the manuscript. occurring isothiocyanates. Chem. Res. Toxicol. 23 (8), 1307-1309. doi: 10.1021/ tx100187f

Dzamitika, S., Salerno, M., Pereira-Maia, E., Le Moyec, L., and Garnier-Suillerot, A. (2006). Preferential energy- and potential-dependent accumulation of cisplatingutathione complexes in human cancer cell lines (GLC4 and K562): A likely role of mitochondria. J. Bioenerg. Biomembr. 38 (1), 11-21. doi: 10.1007/s10863006-9001-x

Elkashty, O. A., Ashry, R., Elghanam, G. A., Pham, H. M., Su, X., Stegen, C., et al. (2018). Broccoli extract improves chemotherapeutic drug efficacy against headneck squamous cell carcinomas. Med. Oncol. 35 (9), 124. doi: 10.1007/s12032018-1186-4

Farzaei, M. H., Bahramsoltani, R., and Rahimi, R. (2016). Phytochemicals as Adjunctive with Conventional Anticancer Therapies. Curr. Pharm. Des. 22 (27), 4201-4218. doi: 10.2174/1381612822666160601100823

Fimognari, C., Nusse, M., Lenzi, M., Sciuscio, D., Cantelli-Forti, G., and Hrelia, P. (2006). Sulforaphane increases the efficacy of doxorubicin in mouse fibroblasts characterized by p53 mutations. Mutat. Res. 601 (1-2), 92-101. doi: 10.1016/ j.mrfmmm.2006.06.001

Fimognari, C., Lenzi, M., Sciuscio, D., Cantelli-Forti, G., and Hrelia, P. (2007), Combination of doxorubicin and sulforaphane for reversing doxorubicinresistant phenotype in mouse fibroblasts with p53Ser220 mutation. Ann. N. Y. Acad. Sci. 1095, 62-69. doi: 10.1196/annals.1397.008

Fimognari, C., Lenzi, M., Sestili, P., Turrini, E., Ferruzzi, L., Hrelia, P., et al. (2012). Sulforaphane potentiates RNA damage induced by different xenobiotics. PloS One 7 (4), e35267. doi: 10.1371/journal.pone.0035267

Galluzzi, L., Senovilla, L., Vitale, I., Michels, J., Martins, I., Kepp, O., et al. (2012). Molecular mechanisms of cisplatin resistance. Oncogene 31 (15), 1869-1883. doi: 10.1038/onc.2011.384

Ghosh, S. (2019). Cisplatin: The first metal based anticancer drug. Bioorg. Chem. 88, 102925. doi: 10.1016/j.bioorg.2019.102925

Guerrero-Beltran, C. E., Calderon-Oliver, M., Martinez-Abundis, E., Tapia, E., Zarco-Marquez, G., Zazueta, C., et al. (2010a). Protective effect of sulforaphane against cisplatin-induced mitochondrial alterations and impairment in the activity of $\mathrm{NAD}(\mathrm{P}) \mathrm{H}$ : quinone oxidoreductase 1 and gamma glutamyl cysteine ligase: studies in mitochondria isolated from rat kidney and in LLC-PK1 cells. Toxicol. Lett. 199 (1), 80-92. doi: 10.1016/j.toxlet.2010.08.009

Guerrero-Beltran, C. E., Calderon-Oliver, M., Tapia, E., Medina-Campos, O. N., Sanchez-Gonzalez, D. J., Martinez-Martinez, C. M., et al. (2010b). Sulforaphane protects against cisplatin-induced nephrotoxicity. Toxicol. Lett. 192 (3), 278-285. doi: 10.1016/j.toxlet.2009.11.007

Guerrero-Beltran, C. E., Mukhopadhyay, P., Horvath, B., Rajesh, M., Tapia, E., Garcia-Torres, I., et al. (2012). Sulforaphane, a natural constituent of broccoli, prevents cell death and inflammation in nephropathy. J. Nutr. Biochem. 23 (5), 494-500. doi: 10.1016/j.jnutbio.2011.02.004

Holditch, S. J., Brown, C. N., Lombardi, A. M., Nguyen, K. N., and Edelstein, C. L. (2019). Recent Advances in Models, Mechanisms, Biomarkers, and Interventions in Cisplatin-Induced Acute Kidney Injury. Int. J. Mol. Sci. 20 (12), E301. doi: 10.3390/ijms20123011

Hu, L., Miao, W., Loignon, M., Kandouz, M., and Batist, G. (2010). Putative chemopreventive molecules can increase Nrf2-regulated cell defense in some 
human cancer cell lines, resulting in resistance to common cytotoxic therapies. Cancer Chemother. Pharmacol. 66 (3), 467-474. doi: 10.1007/s00280-009-1182-7

Hunakova, L., Gronesova, P., Horvathova, E., Chalupa, I., Cholujova, D., Duraj, J., et al. (2014). Modulation of cisplatin sensitivity in human ovarian carcinoma A2780 and SKOV3 cell lines by sulforaphane. Toxicol. Lett. 230 (3), 479-486. doi: 10.1016/j.toxlet.2014.08.018

Kallifatidis, G., Labsch, S., Rausch, V., Mattern, J., Gladkich, J., Moldenhauer, G., et al. (2011). Sulforaphane increases drug-mediated cytotoxicity toward cancer stem-like cells of pancreas and prostate. Mol. Ther. 19 (1), 188-195. doi: $10.1038 / \mathrm{mt} .2010 .216$

Kan, S. F., Wang, J., and Sun, G. X. (2018). Sulforaphane regulates apoptosis- and proliferation related signaling pathways and synergizes with cisplatin to suppress human ovarian cancer. Int. J. Mol. Med. 42 (5), 2447-2458. doi: $10.3892 /$ ijmm.2018.3860

Karasawa, T., and Steyger, P. S. (2015). An integrated view of cisplatin-induced nephrotoxicity and ototoxicity. Toxicol. Lett. 237 (3), 219-227. doi: 10.1016/ j.toxlet.2015.06.012

Kerr, C., Adhikary, G., Grun, D., George, N., and Eckert, R. L. (2018). Combination cisplatin and sulforaphane treatment reduces proliferation, invasion, and tumor formation in epidermal squamous cell carcinoma. Mol. Carcinog. 57 (1), 3-11. doi: 10.1002/mc.22714

Lan, H., Yuan, H., and Lin, C. (2017). Sulforaphane induces p53-deficient SW480 cell apoptosis via the ROS-MAPK signaling pathway. Mol. Med. Rep. 16 (5), 7796-7804. doi: $10.3892 / \mathrm{mmr} .2017 .7558$

Lee, Y. J., and Lee, S. H. (2017). Pro-oxidant activity of sulforaphane and cisplatin potentiates apoptosis and simultaneously promotes autophagy in malignant mesothelioma cells. Mol. Med. Rep. 16 (2), 2133-2141. doi: 10.3892/ mmr.2017.6789

Li, B., Kim, D. S., Yadav, R. K., Kim, H. R., and Chae, H. J. (2015). Sulforaphane prevents doxorubicin-induced oxidative stress and cell death in rat $\mathrm{H} 9 \mathrm{c} 2$ cells. Int. J. Mol. Med. 36 (1), 53-64. doi: 10.3892/ijmm.2015.2199

Li, Q. Q., Xie, Y. K., Wu, Y., Li, L. L., Liu, Y., Miao, X. B., et al. (2017). Sulforaphane inhibits cancer stem-like cell properties and cisplatin resistance through miR214-mediated downregulation of c-MYC in non-small cell lung cancer. Oncotarget 8 (7), 12067-12080. doi: 10.18632/oncotarget.14512

McGowan, J. V., Chung, R., Maulik, A., Piotrowska, I., Walker, J. M., and Yellon, D. M. (2017). Anthracycline Chemotherapy and Cardiotoxicity. Cardiovasc. Drugs Ther. 31 (1), 63-75. doi: 10.1007/s10557-016-6711-0

Mielczarek, L., Krug, P., Mazur, M., Milczarek, M., Chilmonczyk, Z., and Wiktorska, K. (2019). In the triple-negative breast cancer MDA-MB-231 cell line, sulforaphane enhances the intracellular accumulation and anticancer action of doxorubicin encapsulated in liposomes. Int. J. Pharm. 558, 311318. doi: 10.1016/j.ijpharm.2019.01.008

Milkovic, L., Zarkovic, N., and Saso, L. (2017). Controversy about pharmacological modulation of Nrf2 for cancer therapy. Redox Biol. 12, 727-732. doi: 10.1016/ j.redox.2017.04.013

Muz, B., de la Puente, P., Azab, F., and Azab, A. K. (2015). The role of hypoxia in cancer progression, angiogenesis, metastasis, and resistance to therapy. Hypoxia (Auckl) 3, 83-92. doi: 10.2147/HP.S93413

Negrette-Guzman, M. (2019). Combinations of the antioxidants sulforaphane or curcumin and the conventional antineoplastics cisplatin or doxorubicin as prospects for anticancer chemotherapy. Eur. J. Pharmacol. 859, 172513. doi: 10.1016/j.jphar.2019.172513

Pappa, G., Bartsch, H., and Gerhauser, C. (2007). Biphasic modulation of cell proliferation by sulforaphane at physiologically relevant exposure times in a human colon cancer cell line. Mol. Nutr. Food Res. 51 (8), 977-984. doi: 10.1002/mnfr.200700115

Pastorek, M., Simko, V., Takacova, M., Barathova, M., Bartosova, M., Hunakova, L., et al. (2015). Sulforaphane reduces molecular response to hypoxia in ovarian tumor cells independently of their resistance to chemotherapy. Int. J. Oncol. 47 (1), 51-60. doi: 10.3892/ijo.2015.2987

Piberger, A. L., Koberle, B., and Hartwig, A. (2014). The broccoli-born isothiocyanate sulforaphane impairs nucleotide excision repair: XPA as one potential target. Arch. Toxicol. 88 (3), 647-658. doi: 10.1007/s00204-013-1178-2

Rackauskas, R., Zhou, D., Uselis, S., Strupas, K., Herr, I., and Schemmer, P. (2017). Sulforaphane sensitizes human cholangiocarcinoma to cisplatin via the downregulation of anti-apoptotic proteins. Oncol. Rep. 37 (6), 3660-3666. doi: $10.3892 / o r .2017 .5622$
Rafiei, H., Asharafizadeh, M., and Ahmadi, Z. (2020). MicroRNAs as novel target of sulforaphane in cancer therapy: the beginning of a new tale? Phytother. Res., 34 (4), 721-728. doi: 10.1002/ptr6572

Rizzo, V. L., Levine, C. B., and Wakshlag, J. J. (2017). The effects of sulforaphane on canine osteosarcoma proliferation and invasion. Vet. Comp. Oncol. 15 (3), 718-730. doi: $10.1111 /$ vco.12212

Sestili, P., and Fimognari, C. (2015). Cytotoxic and Antitumor Activity of Sulforaphane: The Role of Reactive Oxygen Species. BioMed. Res. Int. 2015, 402386. doi: 10.1155/2015/402386

Sharma, L. K., Lu, J., and Bai, Y. (2009). Mitochondrial respiratory complex I: structure, function and implication in human diseases. Curr. Med. Chem. 16 (10), 1266-1277. doi: 10.2174/092986709787846578

Singh, P., Sharma, R., McElhanon, K., Allen, C. D., Megyesi, J. K., Benes, H., et al. (2015). Sulforaphane protects the heart from doxorubicin-induced toxicity. Free Radic. Biol. Med. 86, 90-101. doi: 10.1016/j.freeradbiomed. 2015.05.028

Tafreshi, N. K., Lloyd, M. C., Bui, M. M., Gillies, R. J., and Morse, D. L. (2014). Carbonic anhydrase IX as an imaging and therapeutic target for tumors and metastases. Subcell. Biochem. 75, 221-254. doi: 10.1007/978-94-007-7359-2_12

Tian, M., Tian, D., Qiao, X., Li, J., and Zhang, L. (2019). Modulation of Mybinduced NF-kB -STAT3 signaling and resulting cisplatin resistance in ovarian cancer by dietary factors. J. Cell Physiol. 234 (11), 21126-21134. doi: 10.1002/ jcp. 28715

Tomlinson, L., Lu, Z. Q., Bentley, R. A., Colley, H. E., Murdoch, C., Webb, S. D., et al. (2019). Attenuation of doxorubicin-induced cardiotoxicity in a human in vitro cardiac model by the induction of the NRF-2 pathway. BioMed. Pharmacother. 112, 108637. doi: 10.1016/j.biopha.2019.108637

Volarevic, V., Djokovic, B., Jankovic, M. G., Harrell, C. R., Fellabaum, C., Djonov, V., et al. (2019). Molecular mechanisms of cisplatin-induced nephrotoxicity: a balance on the knife edge between renoprotection and tumor toxicity. J. BioMed. Sci. 26 (1), 25. doi: 10.1186/s12929-019-0518-9

Wang, X., Li, Y., Dai, Y., Liu, Q., Ning, S., Liu, J., et al. (2016). Sulforaphane improves chemotherapy efficacy by targeting cancer stem cell-like properties via the miR-124/IL-6R/STAT3 axis. Sci. Rep. 6, 36796. doi: 10.1038/srep36796

Xiao, Y., Wang, J., Yan, W., Zhou, Y., Chen, Y., Zhou, K., et al. (2015). Dysregulated miR-124 and miR-200 expression contribute to cholangiocyte proliferation in the cholestatic liver by targeting IL-6/STAT3 signalling. J. Hepatol. 62, 889-896. doi: 10.1016/j.jhep.2014.10.033

Xu, Y., Han, X., Li, Y., Min, H., Zhao, X., Zhang, Y., et al. (2019). Sulforaphane Mediates Glutathione Depletion via Polymeric Nanoparticles to Restore Cisplatin Chemosensitivity. ACS Nano. 13 (11), 13445-13455. doi: 10.1021/ acsnano. $9 \mathrm{~b} 07032$

Yang, F., Wang, F., Liu, Y., Wang, S., Li, X., Huang, Y., et al. (2018). Sulforaphane induces autophagy by inhibition of HDAC6-mediated PTEN activation in triple negative breast cancer cells. Life Sci. 213, 149-157. doi: 10.1016/ j.lfs.2018.10.034

Yang, H., Villani, R. M., Wang, H., Simpson, M. J., Roberts, M. S., Tang, M., et al. (2018). The role of cellular reactive oxygen species in cancer chemotherapy. J. Exp. Clin. Cancer Res. 37 (1), 266. doi: 10.1186/s13046-018-0909-x

Zanichelli, F., Capasso, S., Di Bernardo, G., Cipollaro, M., Pagnotta, E., Carteni, M., et al. (2012). Low concentrations of isothiocyanates protect mesenchymal stem cells from oxidative injuries, while high concentrations exacerbate DNA damage. Apoptosis 17 (9), 964-974. doi: 10.1007/s10495-012-0740-3

Zhang, J., Luo, N., Luo, Y., Peng, Z., Zhang, T., and Li, S. (2012). microRNA-150 inhibits human CD133-positive liver cancer stem cells through negative regulation of the transcription factor c-Myb. Int. J. Oncol. 40 (3), 747-756. doi: $10.3892 /$ ijo. 2011.1242

Conflict of Interest: The authors declare that the research was conducted in the absence of any commercial or financial relationships that could be construed as a potential conflict of interest.

Copyright $\odot 2020$ Calcabrini, Maffei, Turrini and Fimognari. This is an open-access article distributed under the terms of the Creative Commons Attribution License (CC BY). The use, distribution or reproduction in other forums is permitted, provided the original author(s) and the copyright owner(s) are credited and that the original publication in this journal is cited, in accordance with accepted academic practice. No use, distribution or reproduction is permitted which does not comply with these terms. 